\title{
Intermediate Palomar Transient Factory: Realtime Image Subtraction Pipeline
}

\author{
Yi Cao ${ }^{1}$, Peter E. Nugent ${ }^{2,3} \&$ Mansi M. Kasliwal ${ }^{1}$
}

\begin{abstract}
A fast-turnaround pipeline for realtime data reduction plays an essential role in discovering and permitting follow-up observations to young supernovae and fast-evolving transients in modern time-domain surveys. In this paper, we present the realtime image subtraction pipeline in the intermediate Palomar Transient Factory. By using high-performance computing, efficient database, and machine learning algorithms, this pipeline manages to reliably deliver transient candidates within ten minutes of images being taken. Our experience in using high performance computing resources to process big data in astronomy serves as a trailblazer to dealing with data from large-scale timedomain facilities in near future.
\end{abstract}

Subject headings: Surveys - Methods: observational - Supernovae: general

\section{Introduction}

Transients on short timescales (ミ1 day) are becoming particularly interesting for at least two reasons: first, radiation from a supernova (SN) in the first few days of explosion contains rich information about its initial condition, including the progenitor star and its environment (e.g., Kasen 2010; Nakar \& Sari 2010; Rabinak \& Waxman 2011). Hence observations of young SNe open a new phase space to constrain the poorly understood physical properties of the exploding stars in their last evolutionary stages (e.g., Soderberg et al. 2008; Arcavi et al. 2011; Nugent et al. 2011, Cao et al. 2013, 2015, Garnavich et al. 2016). Second, some relativistic transient phenomena have been found to decay on sub-day timescales (e.g., Cenko et al. 2013, 2015). These extreme events are probably related to deaths of the most massive stars.

In recent years, many time-domain projects are motivated to perform fast-cadence transient surveys at different scales, e.g., SDSS (Sako et al. 2008), Pan-STARRS (Rest et al. 2014), the intermediate Palomar Transient Factory (iPTF) and ASAS-SN. Since 2013, as the successor of PTF (Law et al. 2009), iPTF have been performing several fast-cadence experiments to systematically search for and characterize young SNe and fast-evolving transients. Since the survey itself only

\footnotetext{
${ }^{1}$ Astronomy Department, California Institute of Technology, Pasadena, CA 91125, USA

${ }^{2}$ Department of Astronomy, University of California, Berkeley, CA 94720-3411, USA

${ }^{3}$ Lawrence Berkeley National Laboratory, 1 Cyclotron Road, MS 50B-4206, Berkeley, CA 94720, USA
} 
provides single-band monitoring, detailed multi-wavelength follow-up observations are warranted to collect additional information in order to examine the nature of discovered transients, such as ultraviolet-optical-infrared light curves and colors, spectroscopic classification, X-ray and radio observations for non-thermal emission, etc.. As these transients evolve rapidly, to allow time to undertake follow-up observations, we developed an automated image subtraction pipeline that reliably identifies interesting transients soon after the survey images are taken.

This paper is organized as follows: a brief summary of the fast-cadence surveys in iPTF is in \$2. The pipeline and its performance is described in \$3. \$4 summarizes the paper.

\section{A Brief Summary Of iPTF}

iPTF uses the 48 inch Schmidt telescope (P48) at the Palomar Observatory which is equipped with the CFH12K camera (Starr et al. 2000) as the discovery machine to monitor the sky. The CFH12K camera has a mosaic of eleven working CCDs, each of which has $2048 \times 4096$ pixels with a pixel size $1.01^{\prime \prime}$ pixel $^{-1}$. The total field of view is therefore $7.26 \mathrm{deg}^{2}$. The camera has two filter options: Mould $R\left(\lambda_{\text {center }}=6581 \AA\right.$, FWHM $\left.=1251 \AA\right)$ and $g\left(\lambda_{\text {center }}=4754 \AA\right.$, FWHM $=1537 \AA)$. The exposure time is currently chosen to be 60 seconds so a single snapshot reaches a depth of $R \simeq 20.5 \mathrm{mag}$ or $g \simeq 21 \mathrm{mag}$ under the median seeing of $2^{\prime \prime} .0$ at the Palomar Observatory. These limiting magnitudes are set to match our spectroscopic follow-up capability. The mean overhead time of readout and telescope slew for each exposure is about 40 seconds.

In the spring and fall observing seasons, iPTF performs fast-cadence experiments to search for young $\mathrm{SNe}$ and fast transients. Each field is visited multiple times every night in one of the two filters. Once a frame of 11 individual CCD images (file size: $176 \mathrm{MB}$ ) is read out, it is immediately transferred to the National Energy Research Scientific Computing Center (NERSC) for further processing where our realtime image subtraction pipeline is running. iPTF usually takes $\simeq 300$ (in summer) to $>400$ (in winter) exposures every night. So the nightly data volume is $50-70 \mathrm{~GB}$.

\section{Realtime Image Subtraction Pipeline}

A flow chart of the realtime image subtraction pipeline is shown in Figure 1 .

On top of the pipeline is a robotic scheduler. It identifies incoming image frames on the spinning disk, creates a database record for each image frame, and submits one processing job for each frame to the supercomputer queue. It is also responsible for post-processing procedures at the end of a night, including backup of raw and processed data, starting the post-night image stacking procedure, and sending out nightly processing reports.

Each processing job is placed on one computing node with multiple CPUs. It generates a child process for each individual CCD image which utilizes one CPU core and about 4GB of memory. 
With the exception of a serial run for the initial astrometric solution, all CCD images from a given frame are processed in parallel on a computing node. In the following, we describe the major steps in the image subtraction pipeline:

- Image preprocessing - We perform routine procedures of overscan subtraction, bias subtraction and flat-field correction to the raw image. Since bias and flat-field features vary on long timescales, a separate daytime program updates the bias and flat-field images every ten days. For each CCD, this program median-stacks the most recent 100 bias images to make super biases, and the most recent 100 science images in $g$ or $R$ filters to generate super flats in the corresponding filters. Bad-pixel masks are also updated at the same time.

- Astrometric and photometric solvers - Given that each CCD covers $0.57^{\circ} \times 1.15^{\circ}$ sky area, astrometry.net $\mathrm{t}^{1}$ is well suited to derive an initial astrometric solution. The success rate of astrometry.net is $>99 \%$ and the resulting root mean square of the astrometric error is less than half a pixel. However, the current version of astrometry.net does not support parallelization. Running a separate instance of astrometry . net for each CCD child process would require an extremely large mount of memory and could also be limited by the memory access speed. As such we are forced to synchronize all child processes after image preprocessing and run astrometry.net on a single CPU core.

After solving for the astrometric solution, each child process uses SExtractor ${ }^{2}$ to extract a catalog of sources with signal-to-noise ratios (SNRs) $>20$. This catalog is spatially matched to the PTF-iPTF catalog (Ofek et al. 2012b) to calibrate the photometry. To make this step fast, only a median zero-point across each CCD image is calculated, although some CCD images have zero-point variation at different pixels (Ofek et al. 2012a). The precision of the photometric solution at this stage is about 0.1 mag at 20 th magnitude.

- Image registration - In preparation for image subtraction, we need to register both the science image and its corresponding reference image into the same pixel coordinate system. Here the reference image is generated by stacking several best-quality images of the same field. This task is performed by IPAC which is in charge of PTF/iPTF data archiving. Hence, the noise level in the reference image is usually negligible compared to that in the science image. So we remap the reference image into the pixel coordinate system of the science image. First, we generate a catalog of sources with $\mathrm{SNR}>5$ on the science image and a catalog of sources on the reference image. In order to balance the numbers of sources between the science and reference catalogs for more precise alignment, we truncate the reference catalog at the $5 \sigma$ limiting magnitude of the science image. Then the science catalog is matched spatially to the reference catalog with Scamp ${ }^{3}$. Since the reference image always has an astrometric precision

${ }^{1}$ astrometry.net is available at http://astrometry.net.

${ }^{2}$ SExtractor is available at http://www.astromatic.net/software/sextractor

${ }^{3}$ Scamp is available at http://www.astromatic.net/software/scamp 
$\lesssim 0.1^{\prime \prime}$, and since the Scamp matching usually yields a rms $\lesssim 0.1^{\prime \prime}$, the final astrometric solution on the new image is $\lesssim 0.2^{\prime \prime}$. After solving the astrometry of the science image with respect to the reference image, we remap the reference image into the pixel coordinate system of the science image with SWarn 4 .

- Image subtraction - In this step, we subtract the remapped reference image from the science image with HOTPANT: 5 . HOTPANTS is an implementation of the image subtraction algorithm by Alard \& Lupton (1998). In order to minimize the error on the resulting subtraction image, the PSF on the remapped reference image is converted into the PSF on the new image by a PSF convolution kernel. It can be proved in the limiting case of a noise-free reference image that this method is the most optimal solution for background-limited images (Zackay et al. 2016). The convolution kernel is approximated by linear combinations of three Gaussian functions - one of which has a width narrower than the seeing, a second one has a width similar to the seeing, and the third one has a width wider than the seeing. The linear coefficients are calculated by using a list of high-SNR stars which are selected from the SExtractor catalogs and which are evenly distributed across both images. Low spatialfrequency variation of the PSF across the image is also modeled by low-order polynomials in HOTPANTS. Table 3 lists our HOTPANTS input parameters. After image subtraction, the flux level of the subtracted image is normalized to that of the reference image. The subtracted image usually reaches a $5 \sigma$ limiting magnitude of $R \sim 20.5 \mathrm{mag}$ or $g \sim 21 \mathrm{mag}$. After image subtraction, we run SExtractor to look for all sources with $\mathrm{SNR}>5$ as transient candidates. Typically, SExtractor finds a couple of hundred candidates on each subtracted image of an extragalactic field.

- Real-bogus classification - Nonlinearity of the detectors, astrometric misalignment, imperfect convolution kernels, Poisson noise of bright objects, cosmic rays, and many other factors may produce artifacts on the subtracted image. To remove these image-based artifacts, we developed a machine-learning real-bogus classifier which uses the random forest algorithm. For each candidate, we defined 42 features using pixels from new, reference and subtracted images. These features reflect information about the candidate shape, the quality of the subtraction, nearby sources and so on. The "real" training set consists of variable stars, spectroscopically classified supernovae, and asteroids, while the "bogus" set are a large number of randomly selected candidates in our database and confirmed by visual inspection, which represents the whole false positive population. This classifier achieves a $1 \%$ false positive rate at a cost of $5 \%$ false negative rate (Brink et al. 2013, Rebbapragada et al. 2015). In practice, only the top few percent of the candidates in each subtracted image may pass this real-bogus classifier.

\footnotetext{
${ }^{4}$ SWarp is available at http://www.astromatic.net/software/swarp

${ }^{5}$ HOTPANTS is available at http://www . astro.washington.edu/users/becker/v2.0/hotpants.html
} 
- Matching external catalogs - After removing most artifacts in the transient candidate list, the main contaminating sources are real celestial objects, including asteroids, variable stars and active galaxy nuclei (AGNe). As such we further match the transient candidates to the minor planet catalog to remove asteroids. We also use the SDSS catalogs to identify variable stars and AGNe. Outside the SDSS footprint, we built our own star catalog by applying the following criteria to the reference SExtractor catalog:

- MAG_BEST $<20$;

- MU_MAX - MAG_BEST < $0.2+$ Median(MU_MAX - MAG_BEST);

- FWHM_IMAGE $<2 \times$ Median(FWHM_IMAGE);

- ELONGATION $<1.5$

where Median $(*)$ is the median function for the whole SExtractor catalog. This star-galaxy classifier is able to identify $50 \%$ of the field stars and meanwhile misclassifies $5 \%$ of galaxies as stars. Very recently, Miller et al. (in prep.) developed a new random-forest star-galaxy classifier for the PTF/iPTF data. The new classifier uses the whole set of SExtractor outputs and is trained by spectroscopically confirmed stars in SDSS. It achieves a higher true positive rate and a much lower false positive rate.

Matching transient candidates to external catalogs also allows us to assess the quality of a subtracted image by using the following two metrics: the ratio between the total numbers of transient candidates and stars, denoted as $\xi$, and the fraction of transient candidates that pass the real-bogus test (the real-bogus score threshold corresponds to a $1 \%$ false positive rate and $5 \%$ false negative rate), denoted as $\eta$. If we make two reasonable assumptions that the fraction of variable stars is almost constant (denoted as $\phi$ ) at different parts of the sky and that half of the variable stars are brighter in the science image than in the reference image, then $\xi$ should roughly equal to $\phi / 2$. If we further assume that real transients are much rarer than variable stars, then $\eta$ should be close to zero. Subtracted images with large $\xi$ and $\eta$ values usually result from substantial misalignment between the science and reference images.

In addition to point source catalogs, we also spatially associate transient candidates to nearby galaxies in the CLU catalog (Cook et al. in prep.), as the absolute magnitude and environment of a transient also provide valuable information about the nature of the transient.

- Post-night image stacking - Thanks to the multiple visits to the same field each night, after each night, we stack the subtracted images of each field with Swarp to deepen our detection limits by about half a magnitude. These stacked images are used to search for transients slightly below the single-image detection limit, to provide better SNRs for detected transients in single images, and to provide deeper detection limits for non-detection.

In all these steps, the pipeline intensively communicates with our transient database which (1) records metadata of incoming images, (2) searches for reference images, (3) stores metadata 
of subtracted images and transient candidates, and (4) matches transient candidates to external catalogs. In order to facilitate the database, we take the advantage of the NERSC high-performance database service and design a database schema to minimize JOIN operations between big tables.

Table 2 shows the 10th percentile, median, and 90th percentile of elapsed times of the individual steps and the pipeline as a whole. The median total elapsed time of a processing job is 3.1 minutes and its distribution is illustrated in the histogram of Figure 2

We also note that the total elapsed time increases dramatically at low galactic latitudes (Figure 3). This is because the stellar density is high at low galactic latitudes. Any operation involving real celestial sources - such as detecting all sources on an image and matching sources to external catalogs - takes longer at higher stellar densities.

Next, we consider the overhead time of processing images after images have been taken. The data transfer from the Palomar Observatory to NERSC uses a microwave link between the Palomar Observatory and the San Diego Supercomputer Center and ESnet ${ }^{6}$ between the San Diego Supercomputer to NERSC through Caltech. It usually takes 2-3 minutes (Figure 4 ) for the images from the Palomar Observatory to NERSC. There is a weak trend that the data transfer is slightly faster in the second half of the night than in the first half, probably due to decreasing use by people during the early morning hours.

Upon receipt of each image frame, it usually takes up to half a minute for the scheduler to locate this incoming frame. Then the schedule spends seconds on performing a validation check on the image header, including (1) whether the image frame is a science frame, (2) whether it is taken in either the $R$ or $g$ filter, and (3) whether the galactic latitude of the field is above 10 degrees from the Galactic plane. After the image frame passes this validation check, the scheduler generates and submits a job to the supercomputer queue to process it.

The common supercomputer queues are usually filled with jobs from all users and therefore have a queue waiting time ranging from a few minutes to a few hours. To reduce the queue waiting time, NERSC has set up a special realtime queue with eight dedicated computing nodes. Our jobs in this queue are usually executed within half a minute of submission. Occasionally when there are more than eight jobs in the queue, the first eight jobs get executed and other jobs are put on hold for the next available node.

The total wait time between receipt of an image frame and execution of its corresponding process job is shown in Figure 5 .

With the data transfer and wait times included, the total turnaround time from completion of an exposure to candidates with real-bogus scores in the database is shown in Figure 6. As can be seen in the figure, the whole pipeline manages to deliver transient candidates within ten minutes

\footnotetext{
${ }^{6}$ ESnet is the Department of Energy's dedicated science network. More information can be found at https: //www.es.net/
} 
Table 1. HOTPANS parameters

\begin{tabular}{ccc}
\hline \hline Parameter & Value & Note \\
\hline $\mathrm{r}$ & $2.5 \times$ seeing & convolution kernel half width (pixel) \\
$\mathrm{rss}$ & $6 \times$ seeing & half width of substamp to extract around stars (pixel) \\
$\mathrm{tu}$ & recorded in the reference header & upper valid data count of the reference image \\
$\mathrm{tl}$ & median(reference) $-10 \times \sigma_{\text {reference }}{ }^{1}$ & lower valid data count of the reference image \\
iu & 45000 & upper valid data count of the science image \\
il & median(science) $-10 \times \sigma_{\text {science }}{ }^{1}$ & lower valid data count of the science image
\end{tabular}

${ }^{1} \sigma$ denotes the median absolute deviation.

Table 2. Elapsed Times of Pipeline Steps

\begin{tabular}{cccc}
\hline \hline \multirow{2}{*}{ Pipeline Steps } & \multicolumn{3}{c}{ Elapsed Time (seconds) } \\
& 10th percentile & median & 90th percentile \\
\hline Preprocessing & 2.8 & 3.5 & 7.3 \\
Astrometry and photometry solver & 11.9 & 16.3 & 42.2 \\
Image registration & 18.2 & 23.2 & 44.9 \\
Image subtraction & 24.1 & 29.1 & 44.2 \\
Real-bogus classification & 23.8 & 40.7 & 84.3 \\
Matching external catalogs & 28.9 & 61.3 & 168.9 \\
\hline Pipeline total & 125.6 & 190.8 & 379.4 \\
\hline
\end{tabular}


of most images being taken.

Compared to the PTF pipeline, our iPTF pipeline reduced the turnaround time by a factor of $3 \sim 4$. This speedup results from improvements in both hardware and software. For the hardware, we are provided sufficient computing nodes and lustre filesystems with fast I/O. On the software side, we properly designed an interface to the database which records the timing of all steps in the pipeline. These records allow us to locate the bottlenecks of the pipeline and concentrate our man power on them (e.g., indexing the database tables to speed up certain queries).

This fast turnaround pipeline has enabled the iPTF collaboration to discover and study over a hundred young SNe and several fast-evolving transients. The related publications thus far are listed in Table 3 ,

\section{Summary and Discussions}

In this paper, we present the iPTF realtime image subtraction pipeline which, by properly combining high-performance computing resources at NERSC, existing astronomical software, databases, and machine learning algorithm, manages to reliably deliver transient candidates within ten minutes of images being taken at the Palomar Observatory. This pipeline makes identification of interesting fast-evolving transients as fast as possible and saves time for us to undertake follow-up observations. Our experience demonstrates that, in a modern transient survey, data reduction software which follows the survey observations and enables follow-up observations is equally important as the survey and follow-up observations themselves.

A few technical issues in the current pipeline could be improved in future pipelines. First, the pipeline utilizes existing astronomy software which reads input from and passes results onto spinning disks. Therefore, when a large volume of data are being read or written simultaneously, or when the I/O speed of the spinning disks is limited for other reasons, the processing can be substantially slowed down. Furthermore, the I/O speed of the spinning disks is intrinsically much slower than that of memory. Hence, the future pipeline should perform most operations in memory or using BurstBuffer ${ }^{7}$ and limit the communication to the spinning disks when necessary.

Second, the simple Gaussian approximation to the convolution kernel in HOTPANTS is not perfect, because it fails to capture non-Gaussian features of the PSF, especially when the observing conditions are not photometric. In these cases, the image subtraction may produce many subtraction residues of the subtracted images. In order to enhance the quality of image subtraction, several improvements to the Alard \& Lupton algorithm have been proposed and tested (Zackay et al. 2016, Masci et al. in prep.).

\footnotetext{
${ }^{7}$ More information about BurstBuffer can be found at http://www.nersc.gov/users/computational-systems/ cori/burst-buffer/.
} 
Table 3. iPTF publications on young SNe and fast-evolving transients

\begin{tabular}{|c|c|c|}
\hline Objects & Publications & Titles \\
\hline 57 Type II Supernovae & Rubin et al. 2016) & $\begin{array}{l}\text { Type II Supernova Energetics and Comparison of } \\
\text { Light Curves to Shock-cooling Models }\end{array}$ \\
\hline 84 Type II Supernovae & Khazov et al. (2016) & $\begin{array}{l}\text { Flash Spectroscopy: Emission Lines from the Ionized } \\
\text { Circumstellar Material around }<10 \text {-day-old Type II } \\
\text { Supernovae }\end{array}$ \\
\hline iPTF14atg & Cao et al. 2015 & $\begin{array}{l}\text { A strong ultraviolet pulse from a newborn type Ia } \\
\text { supernova }\end{array}$ \\
\hline iPTF13ebh & Hsiao et al. (2015) & $\begin{array}{l}\text { Strong near-infrared carbon in the Type Ia supernova } \\
\text { iPTF13ebh }\end{array}$ \\
\hline iPTF14yb & Cenko et al. 2015) & $\begin{array}{l}\text { iPTF14yb: The First Discovery of a Gamma-Ray Burst } \\
\text { Afterglow Independent of a High-energy Trigger }\end{array}$ \\
\hline iPTF13beo & Gorbikov et al. (2014) & $\begin{array}{l}\text { iPTF13beo: the double-peaked light curve of a Type Ibn } \\
\text { supernova discovered shortly after explosion }\end{array}$ \\
\hline iPTF13ast & Gal-Yam et al. (2014) & $\begin{array}{l}\text { A Wolf-Rayet-like progenitor of SN } 2013 \mathrm{cu} \text { from } \\
\text { spectral observations of a stellar wind }\end{array}$ \\
\hline iPTF13bvn & Fremling et al. 2014) & $\begin{array}{l}\text { The rise and fall of the Type Ib supernova } \\
\text { iPTF13bvn. Not a massive Wolf-Rayet star }\end{array}$ \\
\hline iPTF14jj & Goobar et al. (2014) & The Rise of SN 2014J in the Nearby Galaxy M82 \\
\hline iPTF13bvn & Cao et al. $(2013)$ & $\begin{array}{l}\text { Discovery, Progenitor and Early Evolution of a } \\
\text { Stripped Envelope Supernova iPTF13bvn }\end{array}$ \\
\hline
\end{tabular}


Third, Python is used as a high-level wrapper in the pipeline and also performs simple algebraic calculations. The initialization of Python and execution is generally much slower than a machinelanguage executable. Loading Python modules also highly relies on the I/O speed to the spinning disks. Thus, moving this to the BurstBuffer should also make significant speed-ups.

Moving forward, iPTF will cease operation in February 2017 and make way for the Zwicky Transient Facility (ZTF; Smith et al. 2014; Bellm et al. 2015), which will mount a new camera on the P48 telescope. The new camera will have a field of view of 47 square degrees with faster readout. The optimal survey speed in ZTF will be more than ten times faster than that in iPTF. ZTF will be able to survey the entire visible sky down a depth similar to iPTF every eight hours. Therefore, ZTF is poised to chart the phase space on sub-day timescales. Accordingly, we are organizing a systematic global follow-up network, with the GROWTH (Global Relay of Observatories Watching Transients Happen) program, that is focused on fast transients, young supernovae and asteroids within the first 24 hours of discovery.

Quick discovery pipelines that bridge discovery and rapid follow-up observations will continue to be critical in the ZTF success. The data rate in ZTF will be ten times higher than in iPTF, which is a certain challenge to the ZTF pipeline. By coupling the astronomical pipelines with advanced high-performance computing resources, we are well on our way to delivering this capability for ZTF.

We thank J. Sollerman for useful suggestions to improve the manuscript. YC and PEN acknowledge support from the DOE under grant DE-AC02-05CH11231, Analytical Modeling for Extreme-Scale Computing Environments. YC and MMK also acknowledge support from the National Science Foundation PIRE program grant 1545949. The intermediate Palomar Transient Factory project is a scientific collaboration (PI: S. R. Kulkarni) among the California Institute of Technology, Los Alamos National Laboratory, the University of Wisconsin, Milwaukee, the Oskar Klein Center, the Weizmann Institute of Science, the TANGO Program of the University System of Taiwan, and the Kavli Institute for the Physics and Mathematics of the Universe.

\section{REFERENCES}

Alard, C., \& Lupton, R. H. 1998, ApJ, 503, 325

Arcavi, I., Gal-Yam, A., Yaron, O., et al. 2011, ApJ, 742, L18

Bellm, E. C., Kulkarni, S. R., \& ZTF Collaboration. 2015, in American Astronomical Society Meeting Abstracts, Vol. 225, American Astronomical Society Meeting Abstracts, 328.04

Brink, H., Richards, J. W., Poznanski, D., et al. 2013, MNRAS, 435, 1047

Cao, Y., Kasliwal, M. M., Arcavi, I., et al. 2013, ApJ, 775, L7

Cao, Y., Kulkarni, S. R., Howell, D. A., et al. 2015, Nature, 521, 328 
Cenko, S. B., Kulkarni, S. R., Horesh, A., et al. 2013, ApJ, 769, 130

Cenko, S. B., Urban, A. L., Perley, D. A., et al. 2015, ApJ, 803, L24

Fremling, C., Sollerman, J., Taddia, F., et al. 2014, A\&A, 565, A114

Gal-Yam, A., Arcavi, I., Ofek, E. O., et al. 2014, Nature, 509, 471

Garnavich, P. M., Tucker, B. E., Rest, A., et al. 2016, ApJ, 820, 23

Goobar, A., Johansson, J., Amanullah, R., et al. 2014, ApJ, 784, L12

Gorbikov, E., Gal-Yam, A., Ofek, E. O., et al. 2014, MNRAS, 443, 671

Hsiao, E. Y., Burns, C. R., Contreras, C., et al. 2015, A\&A, 578, A9

Kasen, D. 2010, ApJ, 708, 1025

Khazov, D., Yaron, O., Gal-Yam, A., et al. 2016, ApJ, 818, 3

Law, N. M., Kulkarni, S. R., Dekany, R. G., et al. 2009, PASP, 121, 1395

Nakar, E., \& Sari, R. 2010, ApJ, 725, 904

Nugent, P. E., Sullivan, M., Cenko, S. B., et al. 2011, Nature, 480, 344

Ofek, E. O., Laher, R., Law, N., et al. 2012a, PASP, 124, 62

Ofek, E. O., Laher, R., Surace, J., et al. 2012b, PASP, 124, 854

Rabinak, I., \& Waxman, E. 2011, ApJ, 728, 63

Rebbapragada, U., Bue, B., \& Wozniak, P. R. 2015, in American Astronomical Society Meeting Abstracts, Vol. 225, American Astronomical Society Meeting Abstracts, 434.02

Rest, A., Scolnic, D., Foley, R. J., et al. 2014, ApJ, 795, 44

Rubin, A., Gal-Yam, A., De Cia, A., et al. 2016, ApJ, 820, 33

Sako, M., Bassett, B., Becker, A., et al. 2008, AJ, 135, 348

Smith, R. M., Dekany, R. G., Bebek, C., et al. 2014, in Proc. SPIE, Vol. 9147, Ground-based and Airborne Instrumentation for Astronomy V, 914779

Soderberg, A. M., Berger, E., Page, K. L., et al. 2008, Nature, 453, 469

Starr, B. M., Luppino, G. A., Cuillandre, J.-C., \& Isani, S. 2000, in Proc. SPIE, Vol. 3965, Sensors and Camera Systems for Scientific, Industrial, and Digital Photography Applications, ed. M. M. Blouke, N. Sampat, G. M. Williams, \& T. Yeh, 58-69 
Zackay, B., Ofek, E. O., \& Gal-Yam, A. 2016, ArXiv e-prints, arXiv:1601.02655 


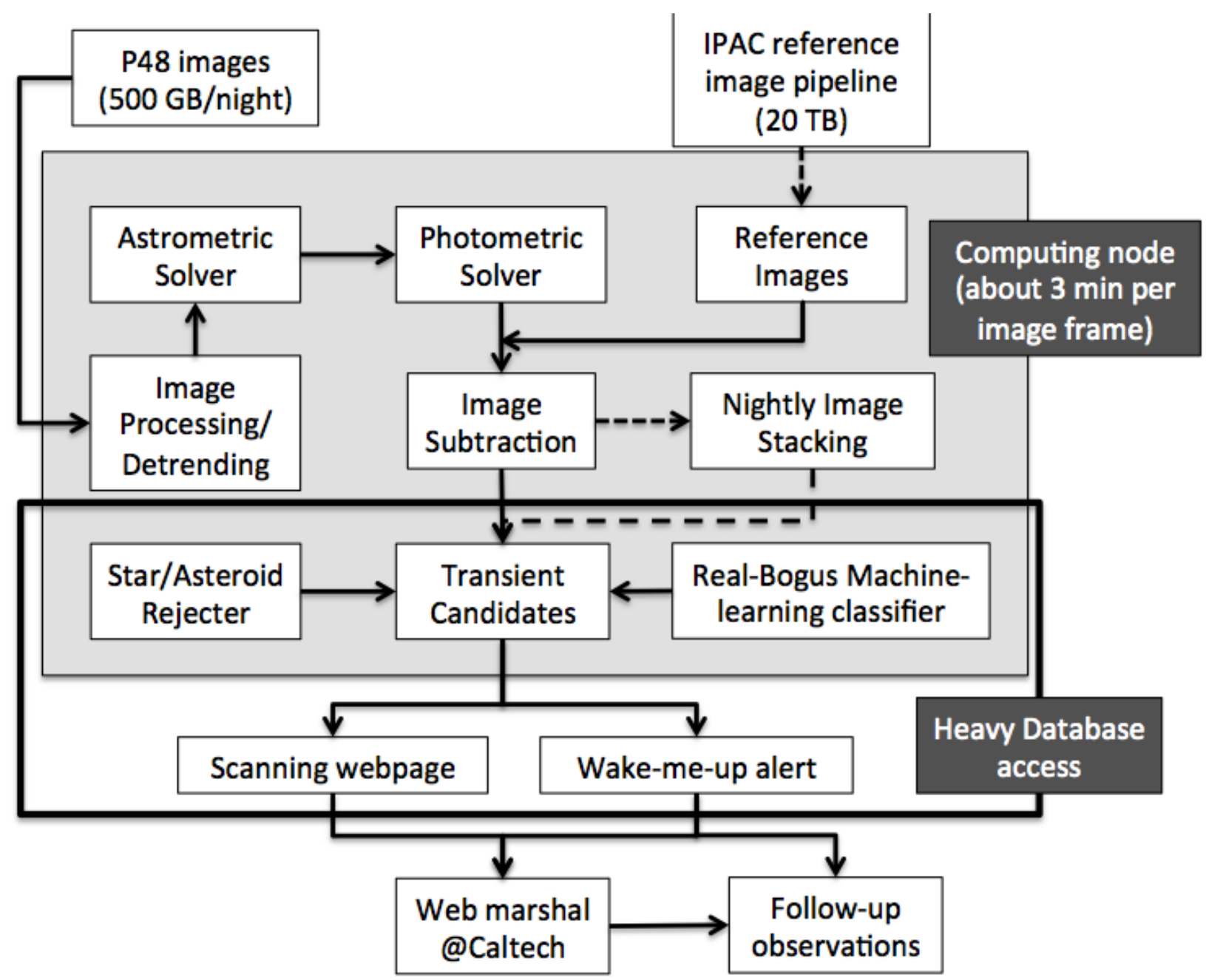

Fig. 1.- Overview of the NERSC image subtraction pipeline. The gray region highlights the realtime pipeline running on the NERSC supercomputer. The black box highlights intercommunication to the database. Solid arrows represent realtime operations and dashed arrows represent post-night operations in the pipeline. This paper focuses on the gray-highlighted region. 


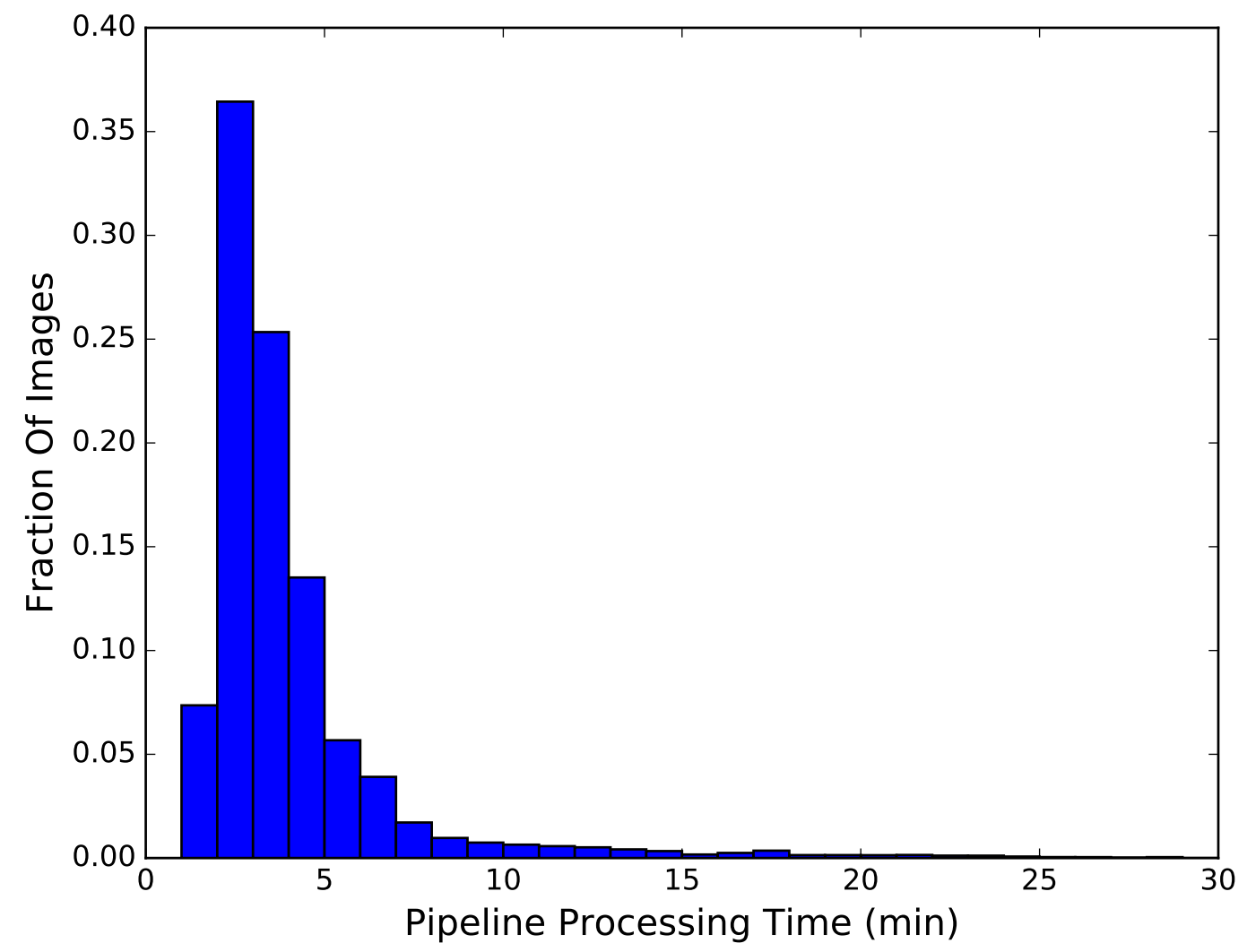

Fig. 2.- Histograms of NERSC pipeline processing time. 


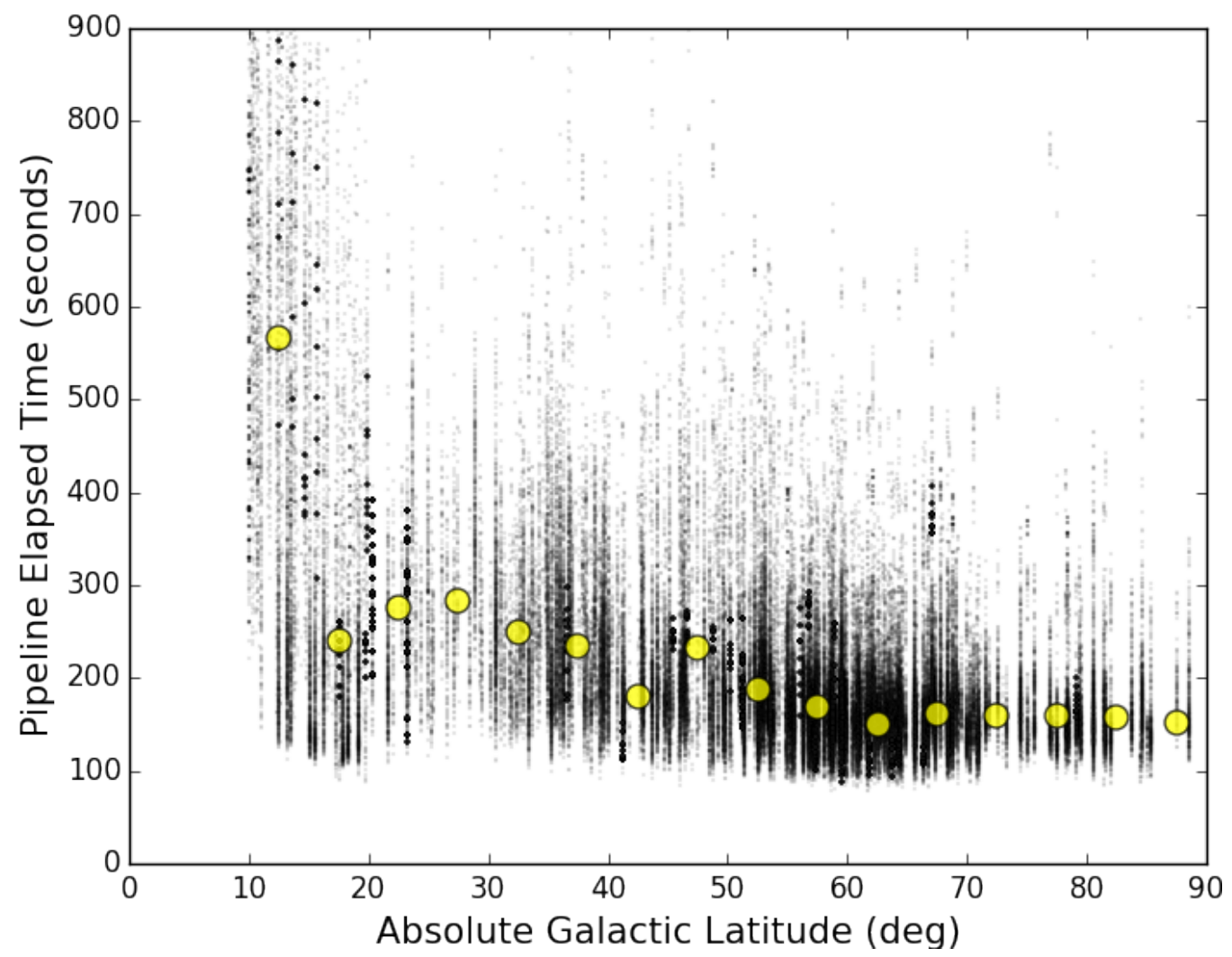

Fig. 3.- Elapsed time of the pipeline as a function of galactic latitude. The gray dots are real measurements and the yellow circles denote the median values in bins of 5 degrees in the absolute galactic latitudes. Note that, since the primary goal of our pipeline is to search for extragalactic transients, it does not process any image within 10 degrees of the Galactic plane. 


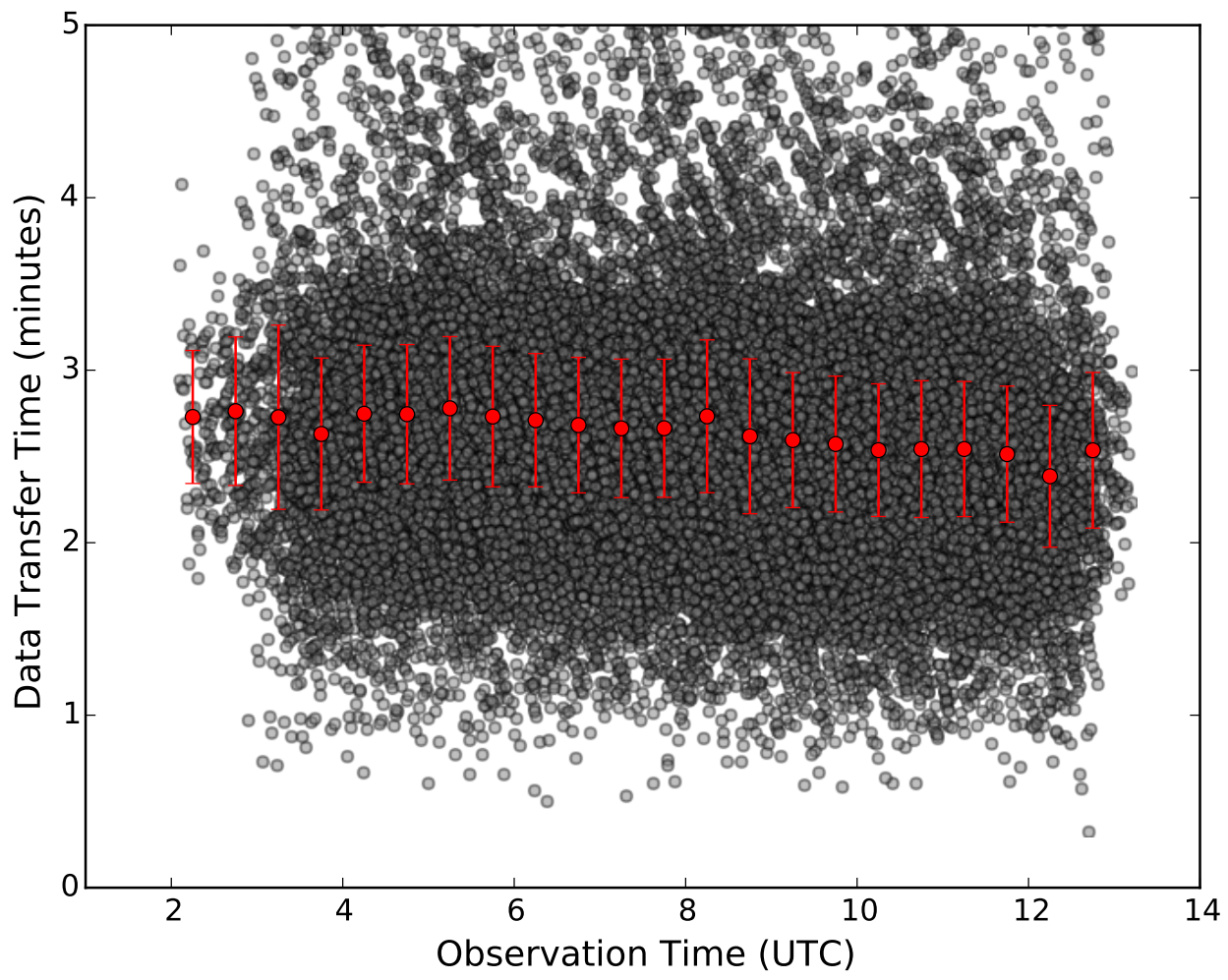

Fig. 4. - The transfer time of individual image frames as a function of UTC hours. The red circles denote the median data transfer time in half-an-hour bins and the red error bars are the median absolute deviation in the corresponding bins. 


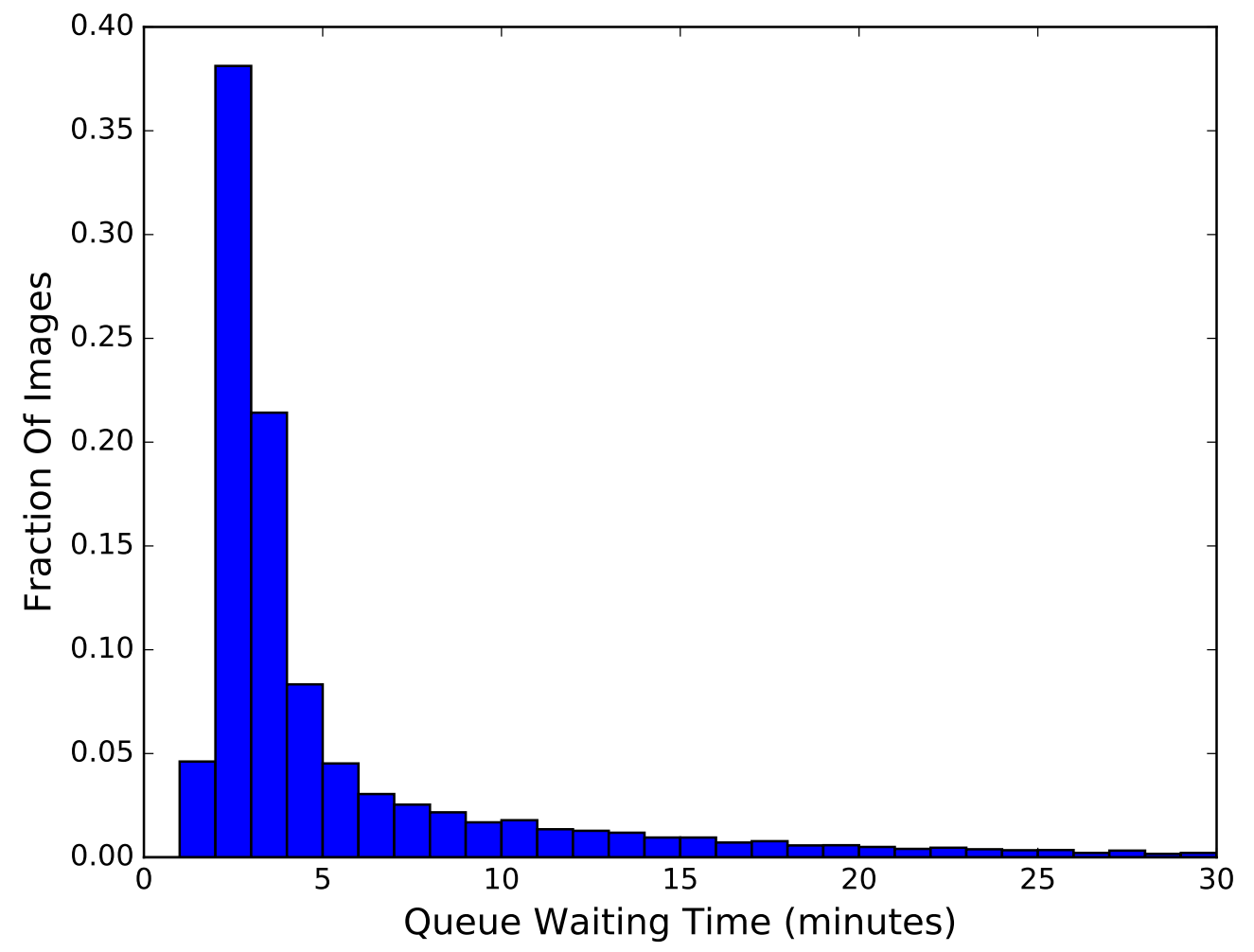

Fig. 5.- The distribution of wait time between receipt of an image frame at NERSC and the execution of its processing job. 


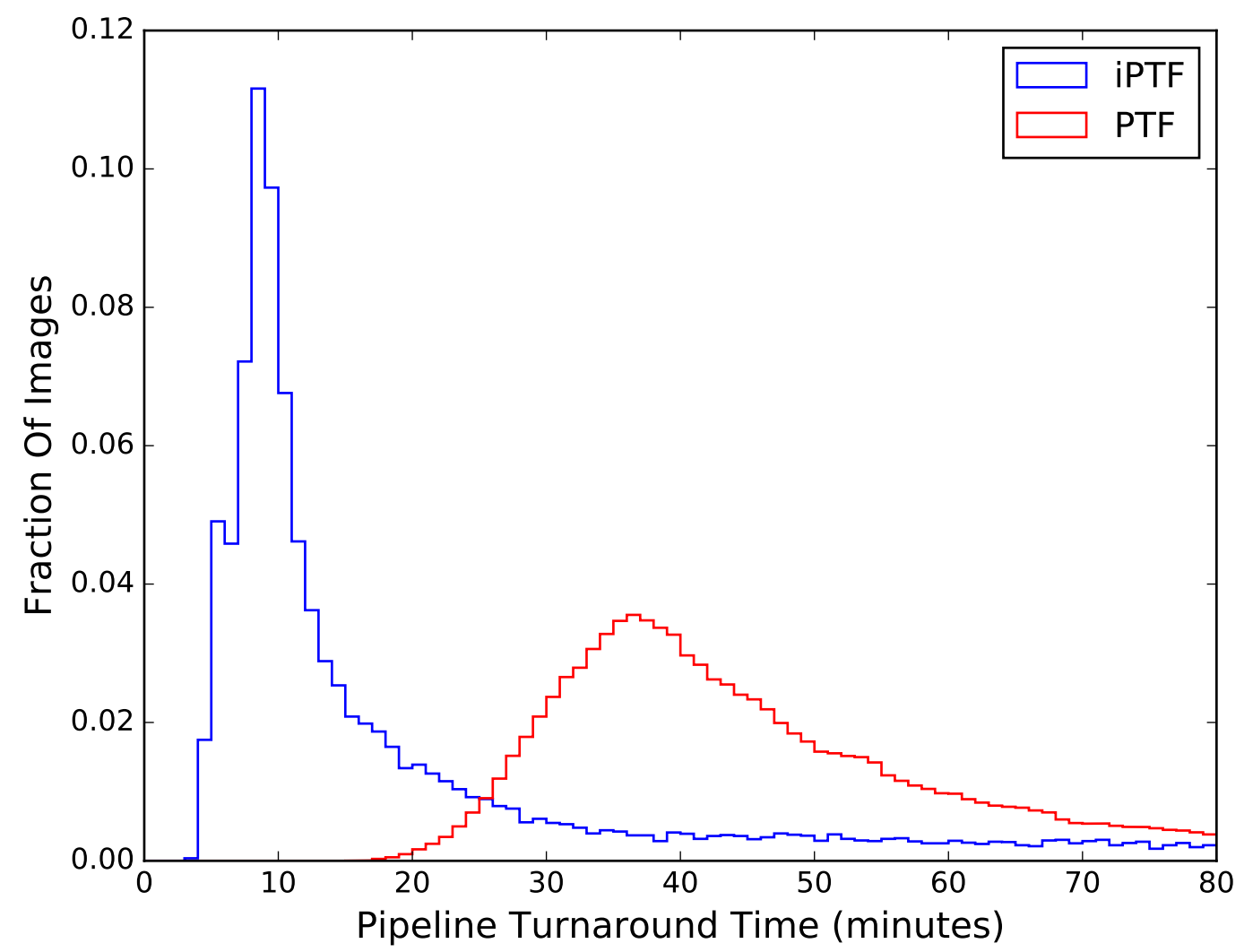

Fig. 6.- Histogram of turnaround time from completion of an exposure to output candidates from the realtime pipeline in PTF (red) and iPTF (blue). 